\title{
Existence of Weak Solutions for Nonlinear Fractional Differential Inclusion with Nonseparated Boundary Conditions
}

\author{
Wen-Xue Zhou ${ }^{1,2}$ and Hai-Zhong Liu ${ }^{1}$ \\ ${ }^{1}$ Department of Mathematics, Lanzhou Jiaotong University, Lanzhou 730070, China \\ ${ }^{2}$ College of Mathematics and Statistics, Xi'an Jiaotong University, Xi'an 710049, China \\ Correspondence should be addressed to Wen-Xue Zhou, wxzhou2006@126.com
}

Received 24 April 2012; Revised 21 July 2012; Accepted 22 July 2012

Academic Editor: Ram N. Mohapatra

Copyright (C) 2012 W.-X. Zhou and H.-Z. Liu. This is an open access article distributed under the Creative Commons Attribution License, which permits unrestricted use, distribution, and reproduction in any medium, provided the original work is properly cited.

We discuss the existence of solutions, under the Pettis integrability assumption, for a class of boundary value problems for fractional differential inclusions involving nonlinear nonseparated boundary conditions. Our analysis relies on the Mönch fixed point theorem combined with the technique of measures of weak noncompactness.

\section{Introduction}

This paper is mainly concerned with the existence results for the following fractional differential inclusion with non-separated boundary conditions:

$$
\begin{gathered}
{ }^{c} D^{\alpha} u(t) \in F(t, u(t)), \quad t \in J:=[0, T], T>0, \\
u(0)=\lambda_{1} u(T)+\mu_{1}, \quad u^{\prime}(0)=\lambda_{2} u^{\prime}(T)+\mu_{2}, \quad \lambda_{1} \neq 1, \lambda_{2} \neq 1,
\end{gathered}
$$

where $1<\alpha \leq 2$ is a real number, ${ }^{c} D^{\alpha}$ is the Caputo fractional derivative. $F: J \times E \rightarrow p(E)$ is a multivalued map, $E$ is a Banach space with the norm $\|\cdot\|$, and $D(E)$ is the family of all nonempty subsets of $E$.

Recently, fractional differential equations have found numerous applications in various fields of physics and engineering [1,2]. It should be noted that most of the books and papers on fractional calculus are devoted to the solvability of initial value problems for differential equations of fractional order. In contrast, the theory of boundary value problems 
for nonlinear fractional differential equations has received attention quite recently and many aspects of this theory need to be explored. For more details and examples, see [3-18] and the references therein.

To investigate the existence of solutions of the problem above, we use Mönch's fixed point theorem combined with the technique of measures of weak noncompactness, which is an important method for seeking solutions of differential equations. This technique was mainly initiated in the monograph of Banaś and Goebel [19] and subsequently developed and used in many papers; see, for example, Banaś and Sadarangani [20], Guo et al. [21], Krzyśka and Kubiaczyk [22], Lakshmikantham and Leela [23], Mönch's [24], O’Regan [25, 26], Szufla $[27,28]$, and the references therein.

In 2007, Ouahab [29] investigated the existence of solutions for $\alpha$-fractional differential inclusions by means of selection theorem together with a fixed point theorem. Very recently, Chang and Nieto [30] established some new existence results for fractional differential inclusions due to fixed point theorem of multivalued maps. Problem (1.1) was discussed for single valued case in the paper [31]; some existence results for single- and multivalued cases for an extension of (1.1) to non-separated integral boundary conditions were obtained in the article [32] and [33]. About other results on fractional differential inclusions, we refer the reader to [34]. As far as we know, there are very few results devoted to weak solutions of nonlinear fractional differential inclusions. Motivated by the above mentioned papers, the purpose of this paper is to establish the existence results for the boundary value problem (1.1) by virtue of the Mönch fixed point theorem combined with the technique of measures of weak noncompactness.

The remainder of this paper is organized as follows. In Section 2, we present some basic definitions and notations about fractional calculus and multivalued maps. In Section 3, we give main results for fractional differential inclusions. In the last section, an example is given to illustrate our main result.

\section{Preliminaries and Lemmas}

In this section, we introduce notation, definitions, and preliminary facts that will be used in the remainder of this paper. Let $E$ be a real Banach space with norm $\|\cdot\|$ and dual space $E^{*}$, and let $(E, \omega)=\left(E, \sigma\left(E, E^{*}\right)\right)$ denote the space $E$ with its weak topology. Here, let $C(J, E)$ be the Banach space of all continuous functions from $J$ to $E$ with the norm

$$
\|y\|_{\infty}=\sup \{\|y(t)\|: 0 \leq t \leq T\},
$$

and let $L^{1}(J, E)$ denote the Banach space of functions $y: J \rightarrow E$ that are the Lebesgue integrable with norm

$$
\|y\|_{L^{1}}=\int_{0}^{T}\|y(t)\| d t
$$

We let $L^{\infty}(J, E)$ to be the Banach space of bounded measurable functions $y: J \rightarrow E$ equipped with the norm

$$
\|y\|_{L^{\infty}}=\inf \{c>0:\|y(t)\| \leq c \text {, a.e. } t \in J\} .
$$


Also, $A C^{1}(J, E)$ will denote the space of functions $y: J \rightarrow E$ that are absolutely continuous and whose first derivative, $y^{\prime}$, is absolutely continuous.

Let $(E,\|\cdot\|)$ be a Banach space, and let $P_{\mathrm{cl}}(E)=\{Y \in D(E): Y$ is closed $\}, P_{b}(E)=$ $\{Y \in D(E): Y$ is bounded $\}, P_{\mathrm{cp}}(E)=\{Y \in D(E): Y$ is compact $\}$, and $P_{\mathrm{cp}, c}(E)=\{Y \in D(E)$ : $Y$ is compact and convex\}. A multivalued map $G: E \rightarrow P(E)$ is convex (closed) valued if $G(x)$ is convex (closed) for all $x \in E$. We say that $G$ is bounded on bounded sets if $G(B)=$ $\cup_{x \in B} G(x)$ is bounded in $E$ for all $B \in P_{b}(E)$ (i.e., $\left.\sup _{x \in B}\{\sup \{\|y\|: y \in G(x)\}\}<\infty\right)$. The mapping $G$ is called upper semicontinuous (u.s.c.) on $E$ if for each $x_{0} \in E$, the set $G\left(x_{0}\right)$ is a nonempty closed subset of $E$ and if for each open set $N$ of $E$ containing $G\left(x_{0}\right)$, there exists an open neighborhood $N_{0}$ of $x_{0}$ such that $G\left(N_{0}\right) \subseteq N$. We say that $G$ is completely continuous if $G(\mathbb{B})$ is relatively compact for every $B \in P_{b}(E)$. If the multivalued map $G$ is completely continuous with nonempty compact values, then $G$ is u.s.c. if and only if $G$ has a closed graph (i.e., $x_{n} \rightarrow x_{*}, y_{n} \rightarrow y_{*}, y_{n} \in G\left(x_{n}\right)$ imply $\left.y_{*} \in G\left(x_{*}\right)\right)$. The mapping $G$ has a fixed point if there is $x \in E$ such that $x \in G(x)$. The set of fixed points of the multivalued operator $G$ will be denoted by Fix $G$. A multivalued map $G: J \rightarrow P_{\mathrm{cl}}(E)$ is said to be measurable if for every $y \in E$, the function

$$
t \longmapsto d(y, G(t))=\inf \{|y-z|: z \in G(t)\}
$$

is measurable. For more details on multivalued maps, see the books of Aubin and Cellina [35], Aubin and Frankowska [36], Deimling [37], Hu and Papageorgiou [38], Kisielewicz [39], and Covitz and Nadler [40].

Moreover, for a given set $V$ of functions $v: J \mapsto \mathbb{R}$, let us denote by $V(t)=\{v(t): v \in$ $V\}, t \in J$, and $V(J)=\{v(t): v \in V, t \in J\}$.

For any $y \in C(J, E)$, let $S_{F, y}$ be the set of selections of $F$ defined by

$$
S_{F, y}=\left\{f \in L^{1}(J, E): f(t) \in F(t, y(t)) \text { a.e. } t \in J\right\} .
$$

Definition 2.1. A function $h: E \rightarrow E$ is said to be weakly sequentially continuous if $h$ takes each weakly convergent sequence in $E$ to a weakly convergent sequence in $E$ (i.e., for any $\left(x_{n}\right)_{n}$ in $E$ with $x_{n}(t) \rightarrow x(t)$ in $(E, \omega)$ then $h\left(x_{n}(t)\right) \rightarrow h(x(t))$ in $(E, \omega)$ for each $\left.t \rightarrow J\right)$.

Definition 2.2. A function $F: Q \rightarrow P_{\mathrm{cl}, \mathrm{cv}}(Q)$ has a weakly sequentially closed graph if for any sequence $\left(x_{n}, y_{n}\right)_{1}^{\infty} \in Q \times Q, y_{n} \in F\left(x_{n}\right)$ for $n \in\{1,2, \ldots\}$ with $x_{n}(t) \rightarrow x(t)$ in $(E, \omega)$ for each $t \in J$ and $y_{n}(t) \rightarrow y(t)$ in $(E, \omega)$ for each $t \in J$, then $y \in F(x)$.

Definition 2.3 (see [41]). The function $x: J \rightarrow E$ is said to be the Pettis integrable on $J$ if and only if there is an element $x_{J} \in E$ corresponding to each $I \subset J$ such that $\varphi\left(x_{I}\right)=\int_{I} \varphi(x(s)) d s$ for all $\varphi \in E^{*}$, where the integral on the right is supposed to exist in the sense of Lebesgue. By definition, $x_{I}=\int_{I} x(s) d s$.

Let $P(J, E)$ be the space of all $E$-valued Pettis integrable functions in the interval $J$.

Lemma 2.4 (see [41]). If $x(\cdot)$ is Pettis' integrable and $h(\cdot)$ is a measurable and essentially bounded real-valued function, then $x(\cdot) h(\cdot)$ is Pettis' integrable. 
Definition 2.5 (see [42]). Let $E$ be a Banach space, $\Omega_{E}$ the set of all bounded subsets of $E$, and $B_{1}$ the unit ball in $E$. The De Blasi measure of weak noncompactness is the map $\beta: \Omega_{E} \rightarrow$ $[0, \infty)$ defined by

$$
\beta(X)=\inf \left\{\epsilon>0 \text { : there exists a weakly compact subset } \Omega \text { of } E \text { such that } X \subset \epsilon B_{1}+\Omega\right\} .
$$

Lemma 2.6 (see [42]). The De Blasi measure of noncompactness satisfies the following properties:

(a) $S \subset T \Rightarrow \beta(S) \leq \beta(T)$;

(b) $\beta(S)=0 \Leftrightarrow S$ is relatively weakly compact;

(c) $\beta(S \cup T)=\max \{\beta(S), \beta(T)\}$;

(d) $\beta\left(\bar{S}^{\omega}\right)=\beta(S)$, where $\bar{S}^{\omega}$ denotes the weak closure of $S$;

(e) $\beta(S+T) \leq \beta(S)+\beta(T)$;

(f) $\beta(a S)=|a| \alpha(S)$;

(g) $\beta(\operatorname{conv}(S))=\beta(S)$;

(h) $\beta\left(\cup_{|\lambda| \leq h} \lambda S\right)=h \beta(S)$.

The following result follows directly from the Hahn-Banach theorem.

Lemma 2.7. Let $E$ be a normed space with $x_{0} \neq 0$. Then there exists $\varphi \in E^{*}$ with $\|\varphi\|=1$ and $\varphi\left(x_{0}\right)=\left\|x_{0}\right\|$.

For completeness, we recall the definitions of the Pettis-integral and the Caputo derivative of fractional order.

Definition 2.8 (see [25]). Let $h: J \rightarrow E$ be a function. The fractional Pettis integral of the function $h$ of order $\alpha \in \mathbb{R}^{+}$is defined by

$$
I^{\alpha} h(t)=\int_{0}^{t} \frac{(t-s)^{\alpha-1}}{\Gamma(\alpha)} h(s) d s
$$

where the sign " $\int$ " denotes the Pettis integral and $\Gamma$ is the gamma function.

Definition 2.9 (see [3]). For a function $h: J \rightarrow E$, the Caputo fractional-order derivative of $h$ is defined by

$$
\left({ }^{c} D_{a+}^{\alpha} h\right)(t)=\frac{1}{\Gamma(n-\alpha)} \int_{a}^{t}(t-s)^{n-\alpha-1} h^{(n)}(s) d s, \quad n-1<\alpha<n,
$$

where $n=[\alpha]+1$ and $[\alpha]$ denotes the integer part of $\alpha$.

Lemma 2.10 (see [43]). Let $E$ be a Banach space with $Q$ a nonempty, bounded, closed, convex, equicontinuous subset of $C(J, E)$. Suppose $F: Q \rightarrow P_{c l, c v}(Q)$ has a weakly sequentially closed graph. If the implication

$$
\bar{V}=\overline{\operatorname{conv}}(\{0\} \cup F(V)) \Longrightarrow V \text { is relatively weakly compact }
$$

holds for every subset $V$ of $Q$, then the operator inclusion $x \in F(x)$ has a solution in $Q$. 


\section{Main Results}

Let us start by defining what we mean by a solution of problem (1.1).

Definition 3.1. A function $y \in A C^{1}(J, E)$ is said to be a solution of (1.1), if there exists a function $v \in L^{1}(J, E)$ with $v(t) \in F(t, y(t))$ for a.e. $t \in J$, such that

$$
{ }^{c} D^{\alpha} y(t)=v(t) \quad \text { a.e. } t \in J, 1<\alpha \leq 2,
$$

and $y$ satisfies conditions $u(0)=\lambda_{1} u(T)+\mu_{1}, u^{\prime}(0)=\lambda_{2} u^{\prime}(T)+\mu_{2}, \lambda_{1} \neq 1, \lambda_{2} \neq 1$.

To prove the main results, we need the following assumptions:

(H1) $F: J \times E \rightarrow P_{\mathrm{cp}, \mathrm{cv}}(E)$ has weakly sequentially closed graph;

(H2) for each continuous $x \in C(J, E)$, there exists a scalarly measurable function $v: J \rightarrow$ $E$ with $v(t) \in F(t, x(t))$ a.e. on $J$ and $v$ is Pettis integrable on $J$;

(H3) there exist $p_{f} \in L^{\infty}\left(J, \mathbb{R}^{+}\right)$and a continuous nondecreasing function $\psi:[0, \infty) \rightarrow$ $[0, \infty)$ such that

$$
\|F(t, u)\|=\sup \{|v|: v \in F(t, u)\} \leq p_{f}(t) \psi(\|u\|)
$$

(H4) for each bounded set $D \subset E$, and each $t \in I$, the following inequality holds:

$$
\beta(F(t, D)) \leq p_{f}(t) \cdot \beta(D)
$$

(H5) there exists a constant $R>0$ such that

$$
\frac{R}{g^{*}+\left\|p_{f}\right\|_{L^{\infty}} \psi(R) G^{*}}>1
$$

where $g^{*}$ and $G^{*}$ are defined by (3.9).

Theorem 3.2. Let E be a Banach space. Assume that hypotheses (H1)-(H5) are satisfied. If

$$
\left\|p_{f}\right\|_{L^{\infty}} G^{*}<1,
$$

then the problem (1.1) has at least one solution on J.

Proof. Let $\rho \in C[0, T]$ be a given function; it is obvious that the boundary value problem [18]

$$
\begin{gathered}
{ }^{c} D^{\alpha} u(t)=\rho(t), \quad t \in(0, T), 1<\alpha \leq 2 \\
u(t)=\lambda_{1} u(T)+\mu_{1}, \quad u^{\prime}(0)=\lambda_{2} u^{\prime}(T)+\mu_{2}, \quad \lambda_{1} \neq 1, \lambda_{2} \neq 1
\end{gathered}
$$


has a unique solution

$$
u(t)=\int_{0}^{T} G(t, s) \rho(s) d s+g(t)
$$

where $G(t, s)$ is defined by the formula

$$
G(t, s)=\left\{\begin{array}{cc}
\frac{(t-s)^{\alpha-1}}{\Gamma(\alpha)}-\frac{\lambda_{1}(T-s)^{\alpha-1}}{\left(\lambda_{1}-1\right) \Gamma(\alpha)}+\frac{\lambda_{2}\left[\lambda_{1} T+\left(1-\lambda_{1}\right) t\right](T-s)^{\alpha-2}}{\left(\lambda_{2}-1\right)\left(\lambda_{1}-1\right) \Gamma(\alpha-1)}, & \text { if } 0 \leq s \leq t \leq T, \\
-\frac{\lambda_{1}(T-s)^{\alpha-1}}{\left(\lambda_{1}-1\right) \Gamma(\alpha)}+\frac{\lambda_{2}\left[\lambda_{1} T+\left(1-\lambda_{1}\right) t\right](T-s)^{\alpha-2}}{\left(\lambda_{2}-1\right)\left(\lambda_{1}-1\right) \Gamma(\alpha-1)}, & \text { if } 0 \leq t \leq s \leq T, \\
g(t)=\frac{\mu_{2}\left[\lambda_{1} T+\left(1-\lambda_{1}\right) t\right]}{\left(\lambda_{2}-1\right)\left(\lambda_{1}-1\right)}-\frac{\mu_{1}}{\lambda_{1}-1} . &
\end{array}\right.
$$

From the expression of $G(t, s)$ and $g(t)$, it is obvious that $G(t, s)$ is continuous on $J \times J$ and $g(t)$ is continuous on $J$. Denote by

$$
G^{*}=\sup \left\{\int_{0}^{T}|G(t, s)| d s, t \in J\right\}, \quad g^{*}=\max _{0 \leq t \leq T}\|g(t)\|
$$

We transform the problem (1.1) into fixed point problem by considering the multivalued operator $N: C(J, E) \rightarrow P_{\mathrm{cl}, \mathrm{cv}}(C(J, E))$ defined by

$$
N(x)=\left\{h \in C(J, E): h(t)=g(t)+\int_{0}^{T} G(t, s) v(s) d s, v \in S_{F, x}\right\},
$$

and refer to [31] for defining the operator $N$. Clearly, the fixed points of $N$ are solutions of Problem (1.1). We first show that (3.10) makes sense. To see this, let $x \in C(J, E)$; by (H2) there exists a Pettis' integrable function $v: J \rightarrow E$ such that $v(t) \in F(t, x(t))$ for a.e. $t \in J$. Since $G(t, \cdot) \in L^{\infty}(J)$, then $G(t, \cdot) v(\cdot)$ is Pettis integrable and thus $N$ is well defined.

Let $R>0$, and consider the set

$$
\begin{gathered}
D=\left\{x \in C(J, E):\|x\|_{\infty} \leq R,\left\|x\left(t_{1}\right)-x\left(t_{2}\right)\right\| \leq\left\|g\left(t_{1}\right)-g\left(t_{2}\right)\right\|\right. \\
\left.+\left\|p_{f}\right\|_{L^{\infty}} \psi(R) \int_{0}^{T}\left\|G\left(t_{2}, s\right)-G\left(t_{1}, s\right)\right\| d s \text { for } t_{1}, t_{2} \in J\right\} ;
\end{gathered}
$$

clearly, the subset $D$ is a closed, convex, bounded, and equicontinuous subset of $C(J, E)$. We shall show that $N$ satisfies the assumptions of Lemma 2.10. The proof will be given in four steps. 
Step 1. We will show that the operator $N(x)$ is convex for each $x \in D$.

Indeed, if $h_{1}$ and $h_{2}$ belong to $N(x)$, then there exists Pettis' integrable functions $v_{1}(t)$, $v_{2}(t) \in F(t, x(t))$ such that, for all $t \in J$, we have

$$
h_{i}(t)=g(t)+\int_{0}^{T} G(t, s) v_{i}(s) d s, \quad i=1,2 .
$$

Let $0 \leq d \leq 1$. Then, for each $t \in J$, we have

$$
\left[d h_{1}+(1-d) h_{2}\right](t)=g(t)+\int_{0}^{T} G(t, s)\left[d v_{1}(s)+(1-d) v_{2}(s)\right] d s .
$$

Since $F$ has convex values, $\left(d v_{1}+(1-d) v_{2}\right)(t) \in F(t, y)$ and we have $d h_{1}+(1-d) h_{2} \in N(x)$.

Step 2. We will show that the operator $N$ maps $D$ into $D$.

To see this, take $u \in N D$. Then there exists $x \in D$ with $u \in N(x)$ and there exists a Pettis integrable function $v: J \rightarrow E$ with $v(t) \in F(t, x(t))$ for a.e. $t \in J$. Without loss of generality, we assume $u(s) \neq 0$ for all $s \in J$. Then, there exists $\varphi_{s} \in E^{*}$ with $\left\|\varphi_{s}\right\|=1$ and $\varphi_{s}(u(s))=\|u(s)\|$. Hence, for each fixed $t \in J$, we have

$$
\begin{aligned}
\|u(t)\| & =\varphi_{t}(u(t))=\varphi_{t}\left(g(t)+\int_{0}^{T} G(t, s) v(s) d s\right) \\
& \leq \varphi_{t}(g(t))+\varphi_{t}\left(\int_{0}^{T} G(t, s) v(s) d s\right) \\
& \leq\|g(t)\|+\int_{0}^{T}\|G(t, s)\| \varphi_{t}(v(s)) d s \\
& \leq g^{*}+G^{*} \psi\left(\|x\|_{\infty}\right)\left\|p_{f}\right\|_{L^{\infty}} .
\end{aligned}
$$

Therefore, by (H5), we have

$$
\|u\|_{\infty} \leq g^{*}+\left\|p_{f}\right\|_{L^{\infty}} G^{*} \psi\left(\|R\|_{\infty}\right) \leq R .
$$

Next suppose $u \in N D$ and $\tau_{1}, \tau_{2} \in J$, with $\tau_{1}<\tau_{2}$ so that $u\left(\tau_{2}\right)-u\left(\tau_{1}\right) \neq 0$. Then, there exists $\varphi \in E^{*}$ such that $\left\|u\left(\tau_{2}\right)-u\left(\tau_{1}\right)\right\|=\varphi\left(u\left(\tau_{2}\right)-u\left(\tau_{1}\right)\right)$. Hence,

$$
\begin{aligned}
\left\|u\left(\tau_{2}\right)-u\left(\tau_{1}\right)\right\| & =\varphi\left(g\left(t_{2}\right)-g\left(t_{1}\right)+\int_{0}^{T}\left[G\left(\tau_{2}, s\right)-G\left(\tau_{1}, s\right)\right] \cdot v(s) d s\right) \\
& \leq \varphi\left(g\left(t_{2}\right)-g\left(t_{1}\right)\right)+\varphi\left(\int_{0}^{T}\left[G\left(\tau_{2}, s\right)-G\left(\tau_{1}, s\right)\right] \cdot v(s) d s\right) \\
& \leq\left\|g\left(t_{2}\right)-g\left(t_{1}\right)\right\|+\int_{0}^{T}\left\|G\left(\tau_{2}, s\right)-G\left(\tau_{1}, s\right)\right\| \cdot\|v(s)\| d s \\
& \leq\left\|g\left(t_{2}\right)-g\left(t_{1}\right)\right\|+\psi(R)\left\|p_{f}\right\|_{L^{\infty}} \int_{0}^{T}\left\|G\left(\tau_{2}, s\right)-G\left(\tau_{1}, s\right)\right\| d s ;
\end{aligned}
$$

this means that $u \in D$. 
Step 3. We will show that the operator $N$ has a weakly sequentially closed graph.

Let $\left(x_{n}, y_{n}\right)_{1}^{\infty}$ be a sequence in $D \times D$ with $x_{n}(t) \rightarrow x(t)$ in $(E, \omega)$ for each $t \in J$, $y_{n}(t) \rightarrow y(t)$ in $(E, \omega)$ for each $t \in J$, and $y_{n} \in N\left(x_{n}\right)$ for $n \in\{1,2, \ldots\}$. We will show that $y \in N x$. By the relation $y_{n} \in N\left(x_{n}\right)$, we mean that there exists $v_{n} \in S_{F, x_{n}}$ such that

$$
y_{n}(t)=g(t)+\int_{0}^{T} G(t, s) v_{n}(s) d s
$$

We must show that there exists $v \in S_{F, x}$ such that, for each $t \in J$,

$$
y(t)=g(t)+\int_{0}^{T} G(t, s) v(s) d s
$$

Since $F(\cdot, \cdot)$ has compact values, there exists a subsequence $v_{n_{m}}$ such that

$$
\begin{gathered}
v_{n_{m}}(\cdot) \longrightarrow v(\cdot) \text { in }(E, \omega) \text { as } m \longrightarrow \infty \\
v_{n_{m}}(t) \in F\left(t, x_{n}(t)\right) \text { a.e. } t \in J .
\end{gathered}
$$

Since $F(t, \cdot)$ has a weakly sequentially closed graph, $v \in F(t, x)$. The Lebesgue dominated convergence theorem for the Pettis integral then implies that for each $\varphi \in E^{*}$,

$$
\varphi\left(y_{n}(t)\right)=\varphi\left(g(t)+\int_{0}^{T} G(t, s) v_{n}(s) d s\right) \longrightarrow \varphi\left(g(t)+\int_{0}^{T} G(t, s) v(s) d s\right)
$$

that is, $y_{n}(t) \rightarrow N x(t)$ in $(E, w)$. Repeating this for each $t \in J$ shows $y(t) \in N x(t)$.

Step 4. The implication (2.9) holds. Now let $V$ be a subset of $D$ such that $V \subset$ $\overline{\operatorname{conv}}(N(V) \cup\{0\})$. Clearly, $V(t) \subset \overline{\operatorname{conv}}(N(V) \cup\{0\})$ for all $t \in J$. Hence, $N V(t) \subset N D(t), t \in$ $J$, is bounded in $P(E)$. 
Since function $g$ is continuous on $J$, the set $\overline{\{g(t), t \in J\}} \subset E$ is compact, so $\beta(g(t))=0$. By assumption ( $\mathrm{H} 4)$ and the properties of the measure $\beta$, we have for each $t \in J$

$$
\begin{aligned}
\beta(N(V)(t)) & =\beta\left\{g(t)+\int_{0}^{T} G(t, s) v(s) d s: v \in S_{F, x}, x \in V, t \in J\right\} \\
& \leq \beta\{g(t): t \in J\}+\beta\left\{\int_{0}^{T} G(t, s) v(s) d s: v \in S_{F, x}, x \in V, t \in J\right\} \\
& \leq \beta\left\{\int_{0}^{T} G(t, s) v(s) d s: v(t) \in F(t, x(t)), x \in V, t \in J\right\} \\
& \leq \int_{0}^{T}\|G(t, s)\| \cdot p_{f}(s) \cdot \beta(V(s)) d s \\
& \leq\left\|p_{f}\right\|_{L^{\infty}} \cdot \int_{0}^{T}\|G(t, s)\| \cdot \beta(V(s)) d s \\
& \leq\left\|p_{f}\right\|_{L^{\infty}} \cdot G^{*} \cdot \int_{0}^{T} \beta(V(s)) d s,
\end{aligned}
$$

which gives

$$
\|v\|_{\infty} \leq\left\|p_{f}\right\|_{L^{\infty}} \cdot\|v\|_{\infty} \cdot G^{*}
$$

This means that

$$
\|v\|_{\infty} \cdot\left[1-\left\|p_{f}\right\|_{L^{\infty}} \cdot G^{*}\right] \leq 0
$$

By (3.5) it follows that $\|v\|_{\infty}=0$; that is, $v=0$ for each $t \in J$, and then $V$ is relatively weakly compact in $E$. In view of Lemma 2.10, we deduce that $N$ has a fixed point which is obviously a solution of Problem (1.1). This completes the proof.

In the sequel we present an example which illustrates Theorem 3.2.

\section{An Example}

Example 4.1. We consider the following partial hyperbolic fractional differential inclusion of the form

$$
\begin{gathered}
\left({ }^{c} D^{\alpha} u_{n}\right)(t) \in \frac{1}{7 e^{t+13}}\left(1+\left|u_{n}(t)\right|\right), \quad t \in J:=[0, T], 1<\alpha \leq 2, \\
u(0)=\lambda_{1} u(T)+\mu_{1}, \quad u^{\prime}(0)=\lambda_{2} u^{\prime}(T)+\mu_{2},
\end{gathered}
$$

Set $T=1, \lambda_{1}=\lambda_{2}=-1, \mu_{1}=\mu_{2}=0$, then $g(t)=0$. So $g^{*}=0$. 
Let

$$
E=l^{1}=\left\{u=\left(u_{1}, u_{2}, \ldots, u_{n}, \ldots\right): \sum_{n=1}^{\infty}\left|u_{n}\right|<\infty\right\}
$$

with the norm

$$
\|u\|_{E}=\sum_{n=1}^{\infty}\left|u_{n}\right|
$$

Set

$$
\begin{gathered}
u=\left(u_{1}, u_{2}, \ldots, u_{n}, \ldots\right), \quad f=\left(f_{1}, f_{2}, \ldots, f_{n}, \ldots\right) \\
f_{n}\left(t, u_{n}\right)=\frac{1}{7 e^{t+13}}\left(1+\left|u_{n}\right|\right), \quad t \in J
\end{gathered}
$$

For each $u_{n} \in \mathbb{R}$ and $t \in J$, we have

$$
\left|f_{n}\left(t, u_{n}\right)\right| \leq \frac{1}{7 e^{t+13}}\left(1+\left|u_{n}\right|\right)
$$

Hence conditions (H1), (H2), and (H3) hold with $p_{f}(t)=1 /\left(7 e^{t+13}\right), t \in J$, and $\psi(u)=$ $1+u, u \in[0, \infty)$. For any bounded set $D \subset l^{1}$, we have

$$
\beta(F(t, D)) \leq \frac{1}{7 e^{t+13}} \cdot \beta(D), \quad \forall t \in J
$$

Hence (H4) is satisfied. From (3.8), we have

$$
G(t, s)= \begin{cases}\frac{(t-s)^{\alpha-1}}{\Gamma(\alpha)}-\frac{(1-s)^{\alpha-1}}{2 \Gamma(\alpha)}+\frac{(1-2 t)(1-s)^{\alpha-2}}{4 \Gamma(\alpha-1)}, & \text { if } 0 \leq s \leq t \leq 1, \\ -\frac{(1-s)^{\alpha-1}}{2 \Gamma(\alpha)}+\frac{(1-2 t)(1-s)^{\alpha-2}}{4 \Gamma(\alpha-1)}, & \text { if } 0 \leq t \leq s \leq 1 .\end{cases}
$$


So, we get

$$
\begin{aligned}
\int_{0}^{1} G(t, s) d s= & \int_{0}^{t} G(t, s) d s+\int_{t}^{1} G(t, s) d s \\
= & \int_{0}^{t}\left[\frac{(t-s)^{\alpha-1}}{\Gamma(\alpha)}-\frac{(1-s)^{\alpha-1}}{2 \Gamma(\alpha)}+\frac{(1-2 t)(1-s)^{\alpha-2}}{4 \Gamma(\alpha-1)}\right] d s \\
& +\int_{t}^{1}\left[-\frac{(1-s)^{\alpha-1}}{2 \Gamma(\alpha)}+\frac{(1-2 t)(1-s)^{\alpha-2}}{4 \Gamma(\alpha-1)}\right] d s \\
= & \frac{4 t^{\alpha}-2}{4 \Gamma(\alpha+1)}+\frac{1-2 t}{4 \Gamma(\alpha)} .
\end{aligned}
$$

A simple computation gives

$$
G^{*}<\frac{1}{4 \Gamma(\alpha)}+\frac{1}{2 \Gamma(\alpha+1)}:=A_{\alpha}
$$

We shall check that condition (3.5) is satisfied. Indeed

$$
\|p\|_{L^{\infty}} G^{*}<\frac{1}{7 e^{13}} A_{\alpha}<1
$$

which is satisfied for some $\alpha \in(1,2]$, and (H5) is satisfied for $R>A_{\alpha} /\left(7 e^{13}-A_{\alpha}\right)$. Then by Theorem 3.2, the problem (4.1) has at least one solution on $J$ for values of $\alpha$ satisfying (4.10).

\section{Acknowledgments}

The first author's work was supported by NNSF of China (11161027), NNSF of China (10901075), and the Key Project of Chinese Ministry of Education (210226). The authors are grateful to the referees for their comments according to which the paper has been revised.

\section{References}

[1] R. Hilfer, Applications of Fractional Calculus in Physics, World Scientific, Singapore, 2000.

[2] J. Sabatier, O. P. Agrawal, and J. A. Tenreiro Machado, Advances in Fractional Calculus, Springer, 2007.

[3] A. A. Kilbas, H. H. Srivastava, and J. J. Trujillo, Theory and Applications of Fractional Differential Equations, Elsevier Science B.V., Amsterdam, The Netherlands, 2006.

[4] V. Lakshmikantham, S. Leela, and J. V. Devi, Theory of Fractional Dynamic Systems, Cambridge Scientific Publishers, Cambridge, UK, 2009.

[5] K. S. Miller and B. Ross, An Introduction to the Fractional Calculus and Fractional Differential Equations, John Wiley \& Sons, New York, NY, USA, 1993.

[6] K. B. Oldham and J. Spanier, The Fractional Calculus, Academic Press, New York, NY, USA, 1974.

[7] I. Podlubny, Fractional Differential Equations, Mathematics in Science and Engineering, Academic Press, New York, NY, USA, 1999.

[8] S. G. Samko, A. A. Kilbas, and O. I. Marichev, Fractional Integrals and Derivatives, Theory and Applications, Gordon and Breach Science Publishers, Yverdon, Switzerland, 1993. 
[9] R. P. Agarwal, M. Benchohra, and S. Hamani, "Boundary value problems for fractional differential equations," Advanced Studies in Contemporary Mathematics, vol. 16, pp. 181-196, 2008.

[10] B. Ahmad and J. J. Nieto, "Existence of solutions for nonlocal boundary value problems of higherorder nonlinear fractional differential equations," Abstract and Applied Analysis, vol. 2009, Article ID 494720, 9 pages, 2009.

[11] Z. Bai and H. Lü, "Positive solutions for boundary value problem of nonlinear fractional differential equation," Journal of Mathematical Analysis and Applications, vol. 311, no. 2, pp. 495-505, 2005.

[12] M. El-Shahed and J. J. Nieto, "Nontrivial solutions for a nonlinear multi-point boundary value problem of fractional order," Computers \& Mathematics with Applications, vol. 59, no. 11, pp. 3438-3443, 2010.

[13] C. F. Li, X. N. Luo, and Y. Zhou, "Existence of positive solutions of the boundary value problem for nonlinear fractional differential equations," Computers \& Mathematics with Applications, vol. 59, no. 3, pp. 1363-1375, 2010.

[14] F. Jiao and Y. Zhou, "Existence of solutions for a class of fractional boundary value problems via critical point theory," Computers \& Mathematics with Applications, vol. 62, no. 3, pp. 1181-1199, 2011.

[15] J. Wang and Y. Zhou, "Analysis of nonlinear fractional control systems in Banach spaces," Nonlinear Analysis. Theory, Methods E Applications A, vol. 74, no. 17, pp. 5929-5942, 2011.

[16] G. Wang, B. Ahmad, and L. Zhang, "Impulsive anti-periodic boundary value problem for nonlinear differential equations of fractional order," Nonlinear Analysis. Theory, Methods E Applications A, vol. 74, no. 3, pp. 792-804, 2011.

[17] W.-X. Zhou and Y.-D. Chu, "Existence of solutions for fractional differential equations with multipoint boundary conditions," Communications in Nonlinear Science and Numerical Simulation, vol. 17, no. 3, pp. 1142-1148, 2012.

[18] W. Zhou, Y. Chang, and H. Liu, "Weak solutions for nonlinear fractional dif-ferential equations in Banach spaces," Discrete Dynamics in Nature and Society, vol. 2012, Article ID 527969, 13 pages, 2012.

[19] J. Banaś and K. Goebel, Measures of Noncompactness in Banach Spaces, vol. 60, Marcel Dekker, New York, NY, USA, 1980.

[20] J. Banaś and K. Sadarangani, "On some measures of noncompactness in the space of continuous functions," Nonlinear Analysis. Theory, Methods \& Applications A, vol. 68, no. 2, pp. 377-383, 2008.

[21] D. Guo, V. Lakshmikantham, and X. Liu, Nonlinear Integral Equations in Abstract Spaces, vol. 373 of Mathematics and its Applications, Kluwer Academic, Dordrecht, The Netherlands, 1996.

[22] S. Krzyśka and I. Kubiaczyk, "On bounded pseudo and weak solutions of a nonlinear differential equation in Banach spaces," Demonstratio Mathematica, vol. 32, no. 2, pp. 323-330, 1999.

[23] V. Lakshmikantham and S. Leela, Nonlinear Differential Equations in Abstract Spaces, vol. 2, Pergamon Press, New York, NY, USA, 1981.

[24] H. Mönch, "Boundary value problems for nonlinear ordinary differential equations of second order in Banach spaces," Nonlinear Analysis, vol. 4, no. 5, pp. 985-999, 1980.

[25] D. O'Regan, "Fixed-point theory for weakly sequentially continuous mappings," Mathematical and Computer Modelling, vol. 27, no. 5, pp. 1-14, 1998.

[26] D. O'Regan, "Weak solutions of ordinary differential equations in Banach spaces," Applied Mathematics Letters, vol. 12, no. 1, pp. 101-105, 1999.

[27] S. Szufla, "On the application of measure of noncompactness to existence theorems," Rendiconti del Seminario Matematico della Università di Padova, vol. 75, pp. 1-14, 1986.

[28] S. Szufla and A. Szukała, "Existence theorems for weak solutions of $n$th order differential equations in Banach spaces," Functiones et Approximatio Commentarii Mathematici, vol. 26, pp. 313-319, 1998, Dedicated to Julian Musielak.

[29] A. Ouahab, "Some results for fractional boundary value problem of differential inclusions," Nonlinear Analysis. Theory, Methods E Applications A, vol. 69, no. 11, pp. 3877-3896, 2008.

[30] Y.-K. Chang and J. J. Nieto, "Some new existence results for fractional differential inclusions with boundary conditions," Mathematical and Computer Modelling, vol. 49, no. 3-4, pp. 605-609, 2009.

[31] B. Ahmad, "New results for boundary value problems of nonlinear fractional differential equations with non-separated boundary conditions," Acta Mathematica Vietnamica, vol. 36, pp. 659-668, 2011.

[32] B. Ahmad, J. J. Nieto, and A. Alsaedi, "Existence and uniqueness of solutions for nonlinear fractional differential equations with non-separated type integral boundary conditions," Acta Mathematica Scientia B, vol. 31, no. 6, pp. 2122-2130, 2011.

[33] B. Ahmad and S. K. Ntouyas, "Nonlinear fractional differential inclusions with anti-periodic typeintegral boundary conditions," Discussiones Mathematicae Differential Inclusions, Control and Optimization. In press. 
[34] J. Henderson and A. Ouahab, "Impulsive differential inclusions with fractional order," Computers $\mathcal{E}$ Mathematics with Applications, vol. 59, no. 3, pp. 1191-1226, 2010.

[35] J.-P. Aubin and A. Cellina, Differential Inclusions, vol. 264, Springer, New York, NY, USA, 1984.

[36] J.-P. Aubin and H. Frankowska, Set-Valued Analysis, vol. 2, Birkhäuser, Boston, Mass, USA, 1990.

[37] K. Deimling, Multivalued Differential Equations, vol. 1, Walter De Gruyter, New York, NY, USA, 1992.

[38] S. Hu and N. S. Papageorgiou, Handbook of Multivalued Analysis, Theory, vol. 1, Kluwer Academic, Dordrecht, The Netherlands, 1997.

[39] M. Kisielewicz, Differential Inclusions and Optimal Control, Kluwer Academic, Dordrecht, The Netherlands, 1991.

[40] H. Covitz and S. B. Nadler Jr., "Multi-valued contraction mappings in generalized metric spaces," Israel Journal of Mathematics, vol. 8, pp. 5-11, 1970.

[41] B. J. Pettis, "On integration in vector spaces," Transactions of the American Mathematical Society, vol. 44, no. 2, pp. 277-304, 1938.

[42] F. S. De Blasi, "On a property of the unit sphere in a Banach space," Bulletin Mathématique de la Société des Sciences Mathématiques de Roumanie, vol. 21, pp. 259-262, 1977.

[43] H. A. H. Salem, A. M. A. El-Sayed, and O. L. Moustafa, "A note on the fractional calculus in Banach spaces," Studia Scientiarum Mathematicarum Hungarica, vol. 42, no. 2, pp. 115-130, 2005. 


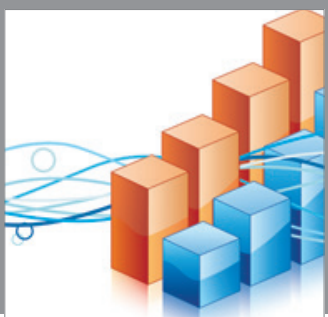

Advances in

Operations Research

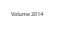

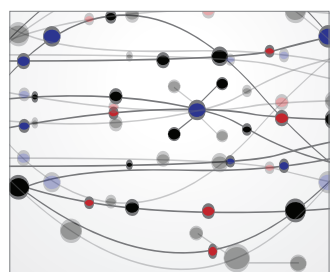

\section{The Scientific} World Journal
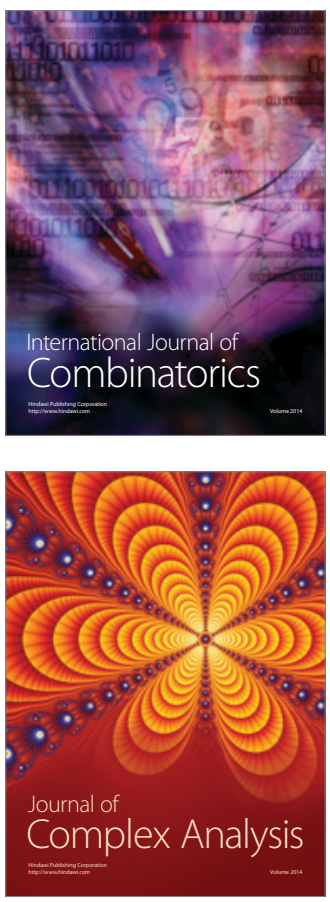

International Journal of

Mathematics and

Mathematical

Sciences
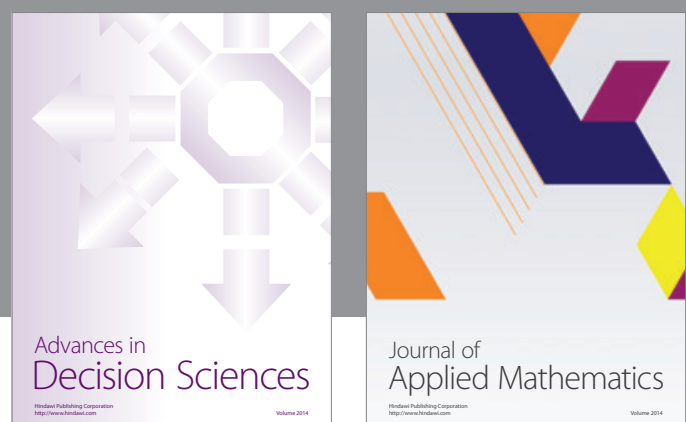

Journal of

Applied Mathematics
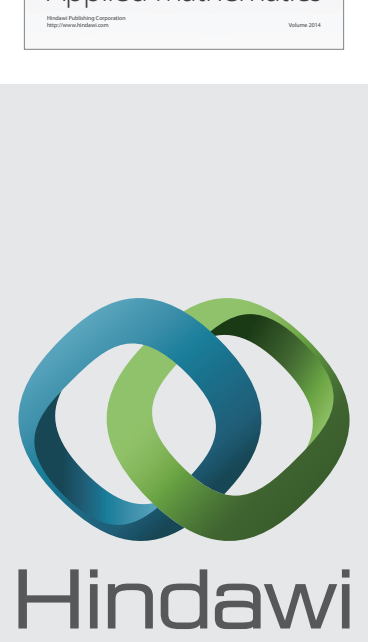

Submit your manuscripts at http://www.hindawi.com
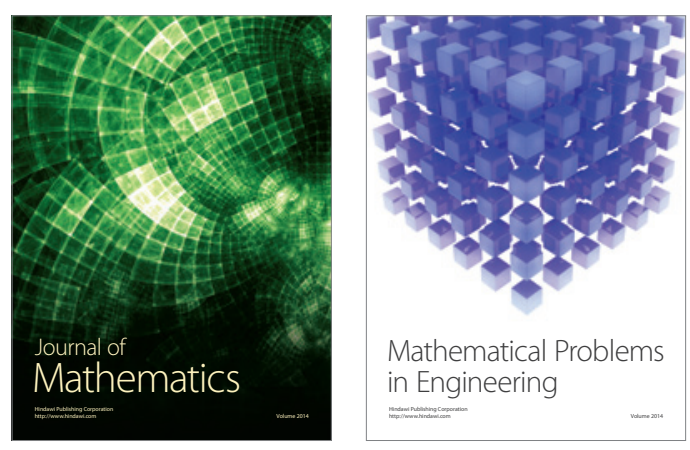

Mathematical Problems in Engineering
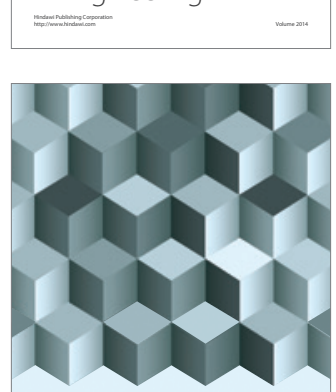

Journal of

Function Spaces
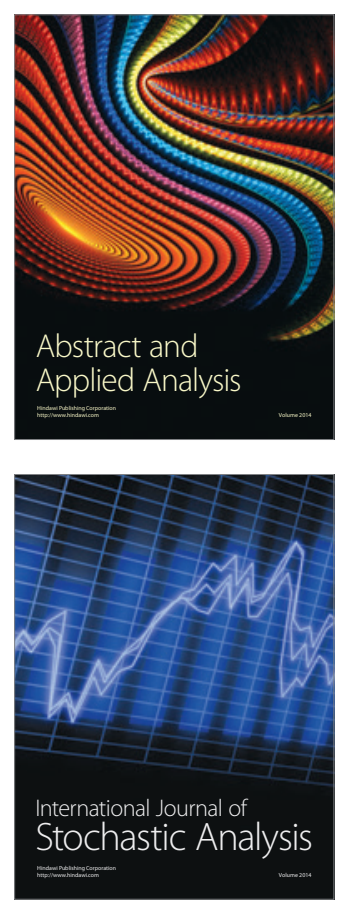

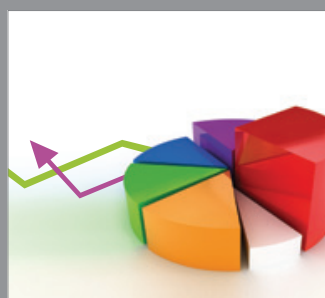

ournal of

Probability and Statistics

Promensencen
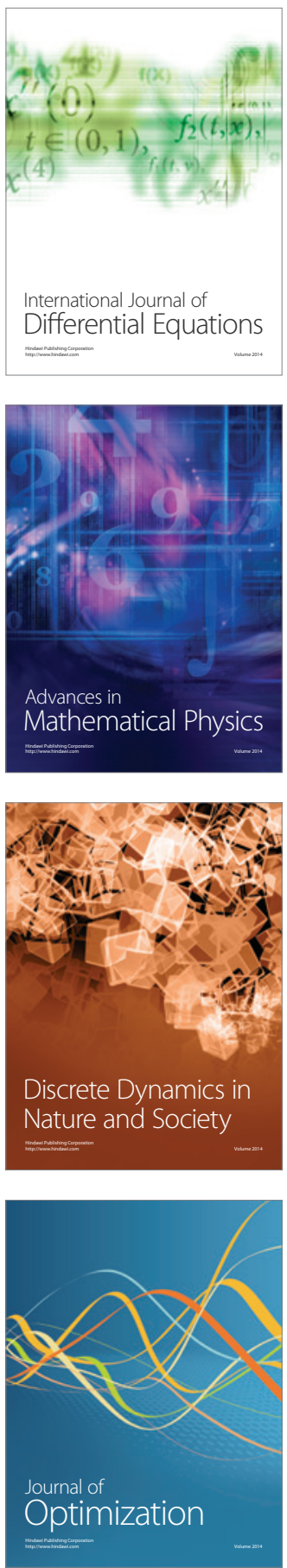